\title{
Aspiration Pneumonia in a Pediatric Patient under General Anesthesia despite Adequate Preoperative Fasting
}

\author{
Sang-Il Yoon, M.D., and Jong-Man Kang, M.D., Ph.D. \\ Department of Anesthesiology and Pain Medicine, Kyung Hee University Hospital at Gangdong, Kyung Hee University College of Medicine, Seoul, Korea
}

\begin{abstract}
Aspiration pneumonia rarely occurs during general anesthesia; however, it can result in fatal pulmonary complications. To reduce aspiration pneumonia, a preoperative fasting time of 8 hours is recommended. A 4-year-old boy with ankyloglossia was scheduled for frenotomy. He completed preoperative fasting time and had no digestive symptoms. Pulmonary aspiration due to unexpected massive vomiting occurred during anesthesia induction. The patient's airway was immediately secured by endotracheal tube. The vomitus in the airway tract was removed by fiberoptic bronchoscopy. Abdomen radiograph taken after this event showed paralytic ileus which can cause aspiration of gastric contents. We describe a case of pneumonia caused by aspiration of gastric contents in a pediatric patient who followed fasting instructions and who was scheduled for outpatient surgery.
\end{abstract}

Key Words: aspiration pneumonia; fasting; general anesthesia.

Preoperative fasting recommendations have been made to reduce the occurrence of pulmonary aspiration. The American Society of Anesthesiologists (ASA) has published practice guidelines for preoperative fasting based on current knowledge.[1] For non-infant patients, a fasting period of six hours after a light meal and eight hours after a meal that includes fried or fatty foods is recommended. Aspiration pneumonia is the most common complication associated with general anesthesia, occurring in approximately 1 out of 3,000 operations; it accounts for up to $9 \%$ of all anesthesia-associated deaths.[2,3] While many reports of pulmonary aspiration by various clinical risk factors have been reported, there were few reports of aspiration pneumonia regarding pediatric patient with no risk factor and importance of preoperative evaluation for preventing aspiration during perioperative period. We describe a pediatric outpatient-surgery case of aspiration pneumonia during anesthesia induction, due to remaining gastric contents, that was successfully treated without any complications.

\section{Case Report}

A male patient aged 4 years and 11 months with ankyloglossia who weighed $18.2 \mathrm{~kg}$ was scheduled for frenotomy; he was

Received on August 6, 2015 Revised on October 1, 2015 Accepted on October 6, 2015

Correspondence to: Jong-Man Kang, Department of Anesthesiology and Pain Medicine, Kyung Hee University Hospital at Gangdong, 892 Dongnam-ro, Gangdong-gu, Seoul 05278, Korea

Tel: +82-2-440-6193, Fax: +82-2-440-7808

E-mail: kjm@khnmc.or.kr

*No potential conflict of interest relevant to this article was reported. admitted on the day of surgery. He had no specific medical history, no allergies to food and drugs and no abnormal results on blood examination, electrocardiography or chest radiography (Fig. 1A). When he was a 3-month-old baby, he was operated for nasal bone fracture at our hospital and no perioperative complications occurred at that time. He had a usual amount for dinner the day before surgery from 7 to 8 

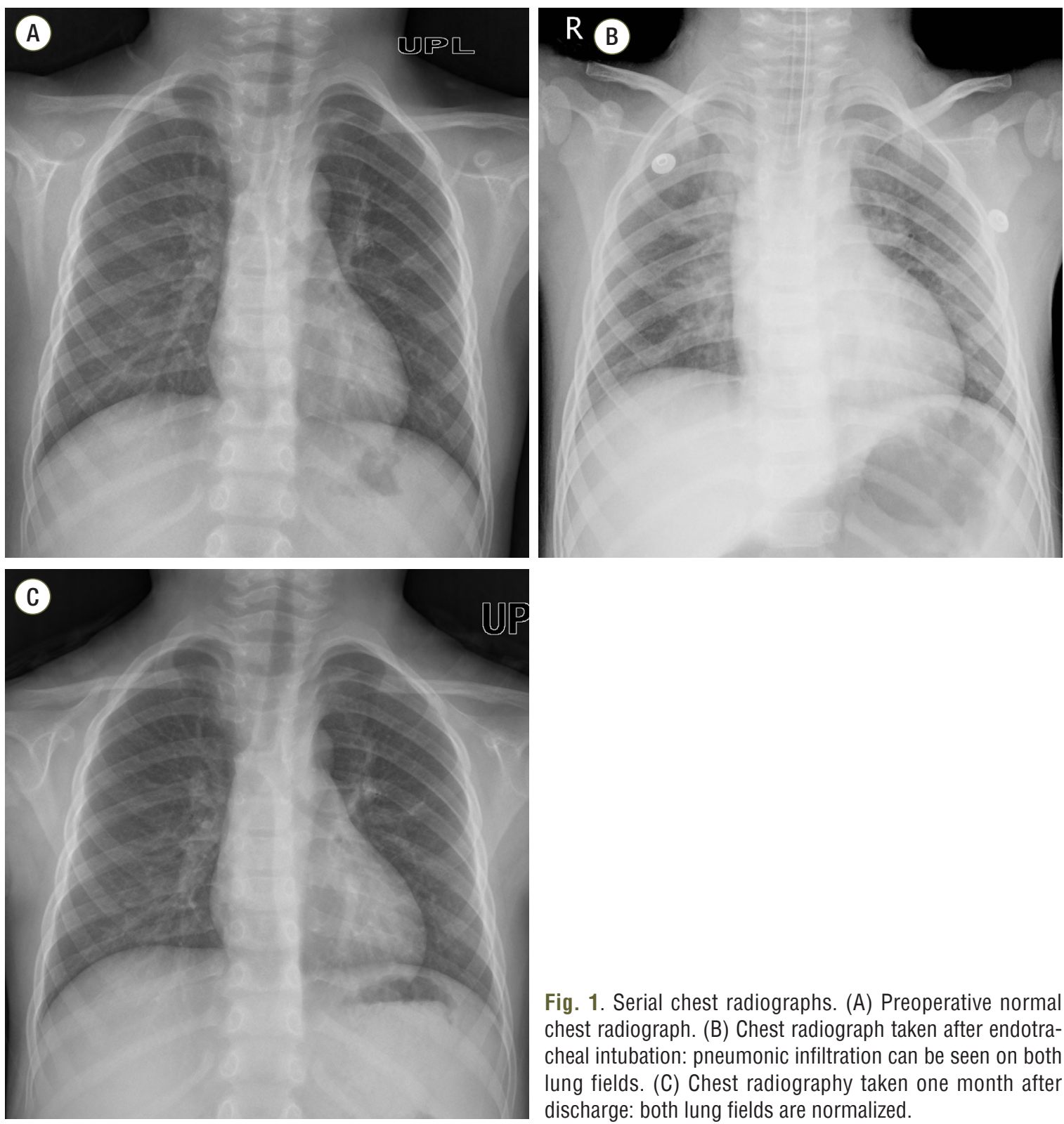

Fig. 1. Serial chest radiographs. (A) Preoperative normal chest radiograph. (B) Chest radiograph taken after endotracheal intubation: pneumonic infiltration can be seen on both lung fields. (C) Chest radiography taken one month after discharge: both lung fields are normalized.

p.m. without any digestive discomfort. He fasted more than 12 hours before surgery.

After completing his preoperative fasting period, which was confirmed by his mother to be more than 8 hours, he was transferred to the operating room at $8 \mathrm{a} . \mathrm{m}$. He had received no premedication. When he entered the operating room without accompanying caregiver, he was not anxious and agitated. The fasting time was verified by his statement once more. He was monitored with a non-invasive blood pressure, pulse oxymetry and electrocardiography. Following induction with $100 \mathrm{mg}$ thiopental sodium intravenous administration and injection of $2 \mathrm{mg}$ cisatracurium, spontaneous breathing ceased. Upon beginning manual ventilation, food secretions and food-like materials began to discharge from the patient's left nostril. He then vomited $200 \mathrm{~mL}$ food he had ingested the day before surgery. The vomitus consisted of rice, kimchi, seaweed and orange and was whitish, pinkish and blackish. Cyanotic changes were observed on the patient's face and his $\mathrm{SpO}_{2}$ level dropped to $60 \%$. The surgical team immediately changed his position from supine to left lateral and head-down and food materials were suctioned from his nose and oral cavity. The airway was imme- 
diately secured with an endotracheal tube and the remaining food materials were removed through that tube. After these actions, his $\mathrm{SpO}_{2}$ levels were remeasured and had increased to $98-100 \%$. Coarse breathing sounds were auscultated in both lung fields. Using fiberoptic bronchoscopy (Bronchofiberscope, LF-DP, Olympus Medical SYSTEMS CORP. LTD, Aomori, Japan), a small volume of the remaining gastric material was removed from the patient's trachea and bronchi and the patient recovered spontaneous breathing. The operation was cancelled and he was transferred to the intensive care unit (ICU) with an endotracheal tube in situ connected to a T-piece ( $50 \%$ oxygen).

The patient had a fever of $38.2^{\circ} \mathrm{C}$, but his body temperature returned to normal after receiving acetaminophen through rectal insertion. Blood examination and radiography were performed immediately as soon as the patient arrived at ICU. The patient's leukocyte counts were 17,100 $/ \mu \mathrm{L}$ (88\% neutrophil segment). Pneumonic infiltrations in both lung fields and paralytic ileus findings were observed on radiography (Fig. 1B and Fig. 2). Antibiotics (cefotaxime and clindamycin) were administered intravenously and a nasogastric tube was inserted. Nebulized normal saline with

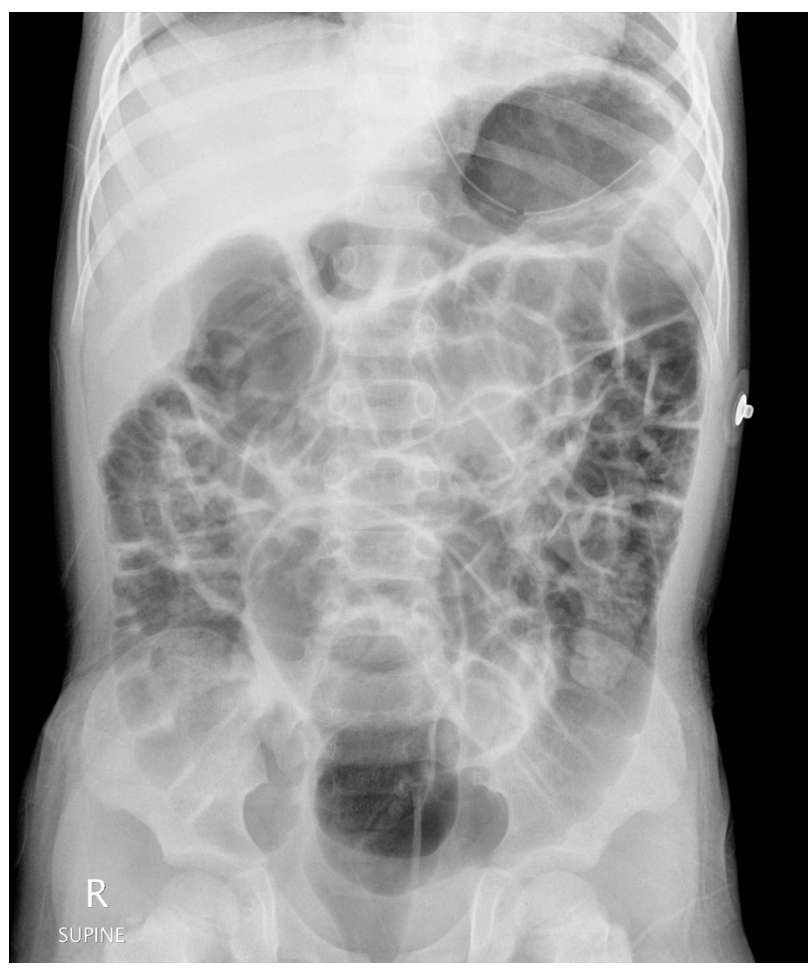

Fig. 2. Abdominal radiography illustrating a paralytic ileus pattern. epinephrine and salbutamol via endotracheal tube for 10 minutes was administered to improve respiratory symptoms. Head-of-bed elevation to avoid gastric regurgitation and help lung expansion, and chest physiotherapy to facilitate clearance of secretions were performed. Therapeutic fasting was applied and total parenteral nutrition with $710 \mathrm{kcal}$ per day was administered for adequate nutritional support. The patient was sedated with intermittent fentanyl injection intravenously when agitated or hyperactive during intubated state. On hospital day (HD) 1, the patient was extubated after showing stable breathing and then $50 \% \mathrm{O}_{2}$ was delivered via a Venturi mask. On HD 2, the patient began to sip water. On HD 3, the patient was allowed a soft diet. He showed no specific symptoms related to digestion and normoactive bowel sounds were auscultated. On follow-up chest radiography, pneumonic infiltrations on the right-middle and lower lobes were improved and the patient was transferred to the general ward. On HD 5, intravenous antibiotics were replaced with oral (cefdinir) and he was discharged with instructions for outpatient follow-up three days later.

On discharge day 2 , he had no digestive or respiratory symptoms except for a brief fever. Chest radiography taken 1 month later revealed no active lung lesions on either lung field (Fig. 1C).

\section{Discussion}

The definition of aspiration is the inhalation of oropharyngeal secretions or gastric contents into the larynx and lower respiratory tract.[4,5] Aspiration pneumonia is regarded as an infectious process because of the inhalation of oropharyngeal secretions that are colonized by pathogenic bacteria and have been intimately related with postoperative mortality or pulmonary morbidity.[4] Warner et al.[6] reported that the overall occurrence of perioperative pulmonary aspiration was 1 in 2,632 patients. Emergency surgeries were related with a greater occurrence of aspiration than elective surgeries. In elective surgeries performed on ASA physical status I and II children, 1 in 8,000 patients revealed pulmonary aspiration. Although the aspiration pneumonia is an uncommon event, this suggests that the possibility of unanticipated events should not be ignored and active prevention should be performed. 
In this pediatric case study, unexpected vomiting with large volume and subsequently undesirable aspiration of gastric contents during anesthesia induction occurred although this pediatric patient with ASA physical status I had no specific medical disease and did not report symptoms such as nausea, vomiting, constipation or any other related digestive disorders before surgery and implemented the fasting intervals.

As presented in abdomen radiography after vomiting, paralytic ileus finding was observed. It may account for aspiration of gastric contents. Paralytic ileus is caused by surgery, severe illness, medication (sedatives and opioids), infection, electrolyte imbalance and stress which lead to release of corticotrophin-releasing factor.[7,8] We speculated that the patient may have preoperative unrecognized chronic or subacute ileus that does not produce related symptoms. This case suggested that paralytic ileus may not always accompany digestive symptoms. There was little possibility of gastric regurgitation caused by increase of airway pressure because vomiting occurred before manual ventilation. If preoperative paralytic ileus is suspected, abdomen radiography can be useful for assessment of digestive function. Emotional and stressful reactions to surgery may influence gastrointestinal motility.

Preoperative fasting is defined as ordered period of time prior to a surgery when patients are not permitted the oral intake of liquids or solids. ASA guidelines recommend that preoperative evaluations include assessment of factors that may increase the risk for aspiration, including gastrointestinal motility disorders, ileus or bowel obstruction, pregnancy, obesity and diabetes mellitus that can influence gastric emptying or fluid volume, potential for difficult airway management, emergency surgery.[1] Separate fasting guidelines are not provided for patients with these or other conditions that may increase the risk of regurgitation or pulmonary aspiration. Also implementing preoperative fasting guidelines cannot guarantee the complete gastric emptying.

To reduce the risk of pulmonary aspiration, preoperative risk evaluation is absolutely demanded. Preoperative risk evaluation is generally based on fasting status and should include close review of medical records, physical examination and patient interview. Pharmacologic therapies to decrease gastric volume and acidity with prokinetics, histamine-2 receptor blockers, proton pump inhibitors, antacids, antiemetics and anticholinergics can be used to decrease the risk of pulmonary aspiration; however, their routine preoperative use is not recommended for patients with no obvious risk factor for pulmonary aspiration.[1] The early use of bronchoscopy for removal of gastric contents in respiratory tract can be helpful to reduce respiratory complication. When massive aspiration of gastric contents occurs, patient should be positioned to left lateral, head down tilt of table applied, prompt oral suctioning performed and airway secured. We should keep in mind the fact that bronchoscopic examination is used for diagnosis and treatment of aspiration pneumonia as first-line approach.

One of the risk factors of pulmonary aspiration is a gastrointestinal motility disorder and the presence of gastric contents. The first step in diagnosing gastroduodenal motility dysfunction is to evaluate gastric emptying.[9] There are various methods for evaluating gastric emptying, but, so far,[10] there is no single method that satisfies all the diverse requirements from clinical and research perspectives.[11] Scintigraphy is largely regarded as the most ideal methods, but it has many drawbacks, including high cost, exposure to harmful radiation, and disagreement regarding appropriate procedural methods across institutions.[9] Ultrasonography is an alternate non-invasive method that requires no radiation exposure and is more commonly available than scintigraphy and a useful tool for gastric contents and volume in many clinical situations when aspiration risk is not easy to identify or determine. $[12,13]$

Treatment of aspiration pneumonia is based on antibiotics and supportive care. Aspiration pneumonia is generally caused by anaerobic oral flora and empiric antibiotic regimens must cover oral anaerobes. Appropriate antibiotic regimens for hospitalized children include ampicillin-sulbactam or clindamycin intravenously.[14] Supportive care includes provision of adequate respiratory support, fluid management, antipyresis, and analgesia. Adequate pain control may keep patient comfortable and encourage coughing which enhances airway clearance. Oxygen supply, mucolytics, and nebulization therapy with salbutamol, budesonide, and epinephrine to improve respiratory symptoms can be used as indicated by clinical conditions.

In conclusion, aspiration pneumonia during general anesthesia is a possible complication among child patients, even those who observed the required preoperative fasting time 
and who have normal digestive function. Therefore, patients with any risk factors for aspiration could be considered for assessments by gastric emptying scintigraphy, ultrasonography, or abdomen radiography if necessary. Also, physical examination of the abdomen by auscultation, inspection and percussion, as well as taking a comprehensive medical history, can be helpful for preventing aspiration. Although prevention is ideal, if pulmonary aspiration does occur during the induction of general anesthesia, it should be managed appropriately as soon as possible.

\section{ORCID}

Sang-II Yoon http://orcid.org/0000-0001-6806-8181

\section{References}

1) American Society of Anesthesiologists Committee: Practice guidelines for preoperative fasting and the use of pharmacologic agents to reduce the risk of pulmonary aspiration: application to healthy patients undergoing elective procedures: an updated report by the American Society of Anesthesiologists Committee on Standards and Practice Parameters. Anesthesiology 2011; 114: 495-511.

2) Shime N, Ono A, Chihara E, Tanaka Y: Current status of pulmonary aspiration associated with general anesthesia: a nationwide survey in Japan. Masui 2005; 54: 1177-85.

3) Lienhart A, Auroy Y, Péquignot F, Benhamou D,
Warszawski J, Bovet M, et al: Survey of anesthesiarelated mortality in France. Anesthesiology 2006; 105: 1087-97.

4) Marik PE: Aspiration pneumonitis and aspiration pneumonia. N Engl J Med 2001;344: 665-71.

5) Marik PE, Kaplan D: Aspiration pneumonia and dysphagia in the elderly. Chest 2003; 124: 328-36.

6) Warner MA, Warner ME, Warner DO, Warner LO, Warner EJ: Perioperative pulmonary aspiration in infants and children. Anesthesiology 1999; 90: 66-71.

7) Saunders MD: Acute colonic pseudo-obstruction. Best Pract Res Clin Gastroenterol 2007; 21: 671-87.

8) Batke M, Cappell MS: Adynamic ileus and acute colonic pseudo-obstruction. Med Clin North Am 2008; 92: 649-70.

9) Camilleri M, Hasler WL, Parkman HP, Quigley EM, Soffer E: Measurement of gastrointestinal motility in the GI laboratory. Gastroenterology 1998; 115: 747-62.

10) Vantrappen G: Methods to study gastric emptying. Dig Dis Sci 1994; 39(12 Suppl): 91S-4S.

11) Smout A, Horowitz M, Armstrong D: Methods to study gastric emptying. Frontiers in gastric emptying. Dig Dis Sci 1994; 39(12 Suppl): 130S-2S.

12) Bolondi L, Bortolotti M, Santi V, Calletti T, Gaiani S, Labò G: Measurement of gastric emptying time by realtime ultrasonography. Gastroenterology 1985; 89: 752-9.

13) Van de Putte P, Perlas A: Ultrasound assessment of gastric content and volume. Br J Anaesth 2014; 113: 12-22.

14) Sandora TJ, Harper MB: Pneumonia in hospitalized children. Pediatr Clin North Am 2005; 52: 1059-81, viii. 